Research Article; Received: March 22, 2021; Accepted: June 29, 2021

\title{
A VARIANT OF THE PROOF OF VAN DER WAERDEN'S THEOREM BY FURSTENBERG
}

\author{
Sadık EYIDOĞAN and Ali Arslan ÖZKURT \\ Department of Mathematics, Cukurova University, Adana, TURKEY
}

\begin{abstract}
Let $R$ be a commutative ring with identity. In this paper, for a given monotone decreasing positive sequence and an increasing sequence of subsets of $R$, we will define a metric on $R$ using them. Then, we will use this kind of metric to obtain a variant of the proof of Van der Waerden's theorem by Furstenberg 3 .
\end{abstract}

\section{INTRODUCTION}

In 1927, Van der Waerden published a famous theorem [5, which states that if the set of positive integers is divided into finitely many classes, then at least one of these classes contains arbitrarily long arithmetic progressions. This theorem was proved by several different methods. The ergodic theoretic method was established by Furstenberg [3] to prove Van der Waerden's theorem. Notion of a dynamical system plays a fundamental role in the proof. A dynamical system is defined as a pair $(X, T)$, where $X$ being a compact metric space and $T$ is a continuous map (homeomorphism) from $X$ into itself. To obtain number theoretic results, a particular kind of a dynamical system, a symbolic system, is preferable. Let $\Lambda=\left\{a_{1}, a_{2}, \ldots, a_{n}\right\}$ be a finite set and form the space $\Omega=\Lambda^{\mathbb{Z}}$ the set of all sequences with entries from $\Lambda$ :

$$
x \in \Omega \Leftrightarrow x=\left\{\ldots, x_{-1}, x_{0}, x_{1}, \ldots\right\} .
$$

Observe that $\Omega$ can be made a compact metric space, with the following metric:

$$
d(x, y)=\inf \left\{\frac{1}{k+1}: x_{i}=y_{i} \text { for }|i|<k\right\} \text { for } x, y \in \Omega .
$$

When $T$ is the shift homeomorphism, that is to say $T x_{n}=x_{n+1}$, from $\Omega$ to itself, the pair $(\Omega, T)$ is called a symbolic dynamical system.

We shall be interested in defining a new symbolic dynamical system in order to

2020 Mathematics Subject Classification. 11B25, 37B10.

Keywords. Van der Waerden theorem, dynamical system, metric space.

@ seyidogan@cu.edu.tr; aozkurt@cu.edu.tr-Corresponding author

(D) 0000-0003-4324-9845; 0000-0001-7631-8435. 
prove Van der Waerden's Theorem. For this purpose, we will consider $\Lambda$ not only as a finite set but also as a ring like $\mathbb{Z}_{n}$, and define a metric using the notion of filtration.

For a comprehensive treatment of the topological results and the ergodic theoretic method described here, the reader is refered to [2] and 4], respectively.

\section{Main Results}

Let $R$ be a commutative ring with identity and

$$
F: F_{1}=\{0\} \subseteq F_{2} \subseteq \cdots \subseteq F_{n} \subseteq \cdots
$$

be a chain of subsets of $R$ such that $R=\bigcup_{i=1}^{\infty} F_{i}$. The chain $F$ is also called a filtration for convenience. For a given monotone decreasing sequence $x:=\left(x_{n}\right)$ on $(0, \infty)$, let's start by defining the function $d_{x, F}: R \times R \longrightarrow[0, \infty)$ as follows:

$$
d_{x, F}(s, t)= \begin{cases}x_{m} & \text { if } A_{s, t} \text { has an upper bound and } m=\max A_{s, t} \\ 0 & \text { otherwise }\end{cases}
$$

where $A_{s, t}=\left\{n \in \mathbb{N}: \forall z \in F_{n} s z=t z\right\}$ and $s, t \in R$.

Proposition 1. $d_{x, F}$ is a metric on $R$.

Proof. (i) It is clear that $d_{x, F}(s, t) \geq 0$ for all $s, t \in R$.

(ii) If $d_{x, F}(s, t)=0$, then $A_{s, t}$ has no upper bound and $1_{R} \in \bigcup_{i=1}^{\infty} F_{i}$ implies $1_{R} \in F_{j}$ for some $j$. It follows from $s 1_{R}=t 1_{R}$ that $s=t$.

(iii) It is easy to see that $d_{x, F}$ is a symmetric function by the definition.

(iv) Let $d_{x, F}(s, t)=x_{m}$. Then $s z=t z$ holds for all $z \in F_{m}$ and there exists an element $y_{n} \in F_{n}$ such that $s y_{n} \neq t y_{n}$ for $n>m$. For a given arbitrary $r \in R$, suppose $d_{x, F}(s, r)=x_{l}<x_{m}$ and $d_{x, F}(r, t)=x_{k}<x_{m}$. Then for each $w \in F_{n}$, where $n=\min \{l, k\}>m$, we have $s w=r w=t w$ which in turn implies that $s$ and $t$ are equal on $F_{n}$. This contradicts with $d_{x, F}(s, t)=x_{m}$. Therefore, $d_{x, F}(s, t) \leq d_{x, F}(s, r)+d_{x, F}(r, t)$ holds for all $s, r, t \in R$.

Remark 1. Suppose that $R$ is an integral domain. For $s, t \in R, s \neq t$, when $s z=t z$ holds, then $z$ must be $0_{R}$ due to the fact that $(s-t) z=0_{R}$ and $R$ has no nonzero zero divisors. In this case, for an arbitrary filtration $F$ :

$$
\begin{gathered}
F_{1}=\{0\} \subseteq F_{2} \subseteq \cdots \subseteq F_{n} \subseteq \cdots \text { such that } R=\bigcup_{i=1}^{\infty} F_{i}, \\
d_{x, F}(s, t)= \begin{cases}x_{1} & \text { if } s \neq t \\
0 & \text { if } s=t .\end{cases}
\end{gathered}
$$

In other words, $d_{x, F}$ must be the discrete metric on integral domains. 
Remark 2. (i) If $x_{n} \leq y_{n}$ for all $n \in \mathbb{N}$, then $d_{x, F} \leq d_{y, F}$.

(ii) If $F$ and $G$ are two filtration such that $F_{n} \subseteq G_{n}$ for all $n \in \mathbb{N}$, then $d_{x, F} \leq$ $d_{x, G}$ holds.

Example 1. For the commutative ring $\mathbb{Z}_{6}$ and the given filtration

$$
F: F_{1}=\{0\} \subseteq\{0,2\} \subseteq\{0,2,4\} \subseteq \mathbb{Z}_{6},
$$

the distance table for a given arbitrary monotone decreasing and positive sequence $x:=\left(x_{n}\right)$ as follows;

\begin{tabular}{c|c|c|c|c|c|c}
$d_{x, F}$ & 0 & 1 & 2 & 3 & 4 & 5 \\
\hline 0 & 0 & $x_{1}$ & $x_{1}$ & $x_{3}$ & $x_{1}$ & $x_{1}$ \\
1 & $x_{1}$ & 0 & $x_{1}$ & $x_{1}$ & $x_{3}$ & $x_{1}$ \\
2 & $x_{1}$ & $x_{1}$ & 0 & $x_{1}$ & $x_{1}$ & $x_{3}$ \\
3 & $x_{3}$ & $x_{1}$ & $x_{1}$ & 0 & $x_{1}$ & $x_{1}$ \\
4 & $x_{1}$ & $x_{3}$ & $x_{1}$ & $x_{1}$ & 0 & $x_{1}$ \\
5 & $x_{1}$ & $x_{1}$ & $x_{3}$ & $x_{1}$ & $x_{1}$ & 0 \\
\hline
\end{tabular}

Theorem 1. Suppose that $R$ is a commutative ring with identity and $\tau$ is a topology on $R$. Then $\tau$ is the discrete topology if and only if there exist a monotone decreasing and positive sequence $x:=\left(x_{n}\right)$ and a filtration $F$ such that $\tau=\tau_{d_{x, F}}$ where $\tau_{d_{x, F}}$ is the metric topology induced by $d_{x, F}$.

Proof. Let $\tau$ be the discrete topology on $R$. If we choose $F$ to be $F_{1}=\{0\} \subseteq F_{2}=R$ and $x$ to be an arbitrary monotone decreasing sequence on $(0, \infty)$, then we get the following metric

$$
d_{x, F}(s, t)= \begin{cases}x_{1} & \text { if } s \neq t \\ 0 & \text { if } s=t\end{cases}
$$

on $R$. As $d_{x, F}$ is a discrete metric, we obtain that $\tau=\tau_{d_{x, F}}$.

Conversely, for a given topology $\tau_{d_{x, F}}$ on $R$, we will show that $\tau_{d_{x, F}}$ is the discrete topology. Since $1_{R} \in R=\bigcup_{i=1}^{\infty} F_{i}$, there is some $k \geq 1$ such that $1_{R} \in F_{k}$. It follows from $1_{R} \in F_{k}$ that $m=\max A_{s, t}<k$ for all $s, t \in R, s \neq t$. Therefore, if we choose $\varepsilon$ from the interval $\left(0, x_{k}\right)$, we get that the open ball of radius $\varepsilon$ centred at $s$ is a singleton, it means that

$$
B(s, \varepsilon)=\left\{r \in R: d_{x, F}(s, r)<\varepsilon\right\}=\{s\} .
$$

Hence, $\tau_{d_{x, F}}$ is the discrete topology on $R$.

It was shown that using the metric $d_{x, F}$ on a commutative ring with identity, we cannot go beyond discrete topology. Therefore, it is necessary to change the definition to get effective results.

Let $R$ be a commutative ring with identity and

$$
F: F_{1}=\{0\} \subseteq F_{2} \subseteq \cdots \subseteq F_{n} \subseteq \cdots
$$


be a chain of subsets of $R$ which do not contain any units. For a given monotone decreasing sequence $x:=\left(x_{n}\right)$ on $(0, \infty)$ that converges to zero, let us define $d_{x, F}$ : $R \times R \longrightarrow[0, \infty)$ as follows:

$$
d_{x, F}(s, t)= \begin{cases}x_{m} & \text { if } A_{s, t} \text { has an upper bound and } m=\max A_{s, t} \\ 0 & \text { otherwise }\end{cases}
$$

where $A_{s, t}=\left\{n \in \mathbb{N}: \forall z \in F_{n} s z=t z\right\}$ and $s, t \in R$.

This time $d_{x, F}$ is a pseudometric on $R$. For this reason, define the space $R^{*}=R / \sim$ of equivalence classes by setting

$$
s \sim t \Leftrightarrow d_{x, F}(s, t)=0
$$

to obtain a metric.

Proposition 2. $d_{x, F}^{*}([s],[t])=d_{x, F}(s, t)$ is a metric on $R^{*}$.

Example 2. (i) Since $\mathbb{Z}_{2}$ has no zero divisors, it is easy to see $d_{x, F}^{*}=d_{x, F}$ for $\mathbb{Z}_{2}^{\mathbb{N}}$ and the filtration

$$
F: F_{0}=\{\theta\} \subseteq F_{1} \subseteq \cdots \subseteq F_{n} \subseteq \cdots
$$

where $F_{n}=\underbrace{\mathbb{Z}_{2} \times \cdots \times \mathbb{Z}_{2}}_{n \text {-times }} \times\{0\} \times \cdots$ for each $n \in \mathbb{N}$ and $\theta=(0,0, \ldots)$.

(ii) For the commutative ring $\mathbb{Z}_{6}^{\mathbb{N}}$ and the filtration

$$
\begin{gathered}
F: F_{0}=\{\theta\} \subseteq F_{1} \subseteq \cdots \subseteq F_{n} \subseteq \cdots \\
\text { where } F_{n}=\underbrace{\{0,2\} \times \cdots \times\{0,2\}}_{n-\text { times }} \times\{0\} \times \cdots \text { for each } n \in \mathbb{N}, \\
\text { we have }[(1,1, \ldots)]=[(4,4, \ldots)] \text { since } d_{x, F}((1,1, \ldots),(4,4, \ldots))=0 \text { holds. }
\end{gathered}
$$

Proposition 3. The operations induced by + and $\cdot$ are continuous on the metric space $\left(\left(\mathbb{Z}_{n}^{\mathbb{N}}\right)^{*}, d_{x, F}^{*}\right)$.

Proof. (i) $+:\left(\mathbb{Z}_{n}^{\mathbb{N}}\right)^{*} \times\left(\mathbb{Z}_{n}^{\mathbb{N}}\right)^{*} \rightarrow\left(\mathbb{Z}_{n}^{\mathbb{N}}\right)^{*},([a],[b]) \mapsto\left[\left(a_{n}+b_{n}\right)_{n \in \mathbb{N}}\right]$

Suppose that $\left(a_{n}, b_{n}\right)$ converges to $(a, b)$. Since $d_{x, F}\left(a_{n}, a\right)+d_{x, F}\left(b_{n}, b\right) \rightarrow 0$, for each $\varepsilon>0$ there exists a natural number $m$ such that for every natural number $n>m$, we have $d_{x, F}\left(a_{n}, a\right)+d_{x, F}\left(b_{n}, b\right) \leq x_{k}<\varepsilon$ where $x_{k}$ is the greatest term of the sequence satisfying the inequality. Therefore, we get $a_{n} z=a z$ and $b_{n} z=b z$ for all $z \in F_{k}$ which imply $\left(a_{n}+b_{n}\right) z=(a+b) z$ and $d_{x, F}\left(a_{n}+b_{n}, a+b\right) \leq x_{k}<\varepsilon$. Hence, $d_{x, F}^{*}\left(\left[a_{n}\right]+\left[b_{n}\right],[a]+[b]\right) \rightarrow 0$ and + is continuous.

(ii) $\cdot:\left(\mathbb{Z}_{n}^{\mathbb{N}}\right)^{*} \times\left(\mathbb{Z}_{n}^{\mathbb{N}}\right)^{*} \rightarrow\left(\mathbb{Z}_{n}^{\mathbb{N}}\right)^{*},([a],[b]) \mapsto\left[\left(a_{n} \cdot b_{n}\right)_{n \in \mathbb{N}}\right]$

First, we show the compatibility of multiplication; for any $s, s_{1}, t, t_{1} \in\left(\mathbb{Z}_{n}^{\mathbb{N}}\right)^{*}$,

$$
s \sim s_{1}, t \sim t_{1} \Rightarrow s t \sim s_{1} t_{1} .
$$


Suppose that $s \sim s_{1}, t \sim t_{1}$. For any $z \in \bigcup_{n=1}^{\infty} F_{n}$, we have

$$
t z=t_{1} z \Leftrightarrow\left(t-t_{1}\right) z=0
$$

since $t \sim t_{1}$. Combining the commutative property of multiplication and $s \sim s_{1}$ that we obtain

$$
s\left(\left(t-t_{1}\right) z\right)=0 \Leftrightarrow(s t) z=s\left(t_{1} z\right)=t_{1}(s z)=\left(t_{1} s_{1}\right) z=\left(s_{1} t_{1}\right) z .
$$

Hence, st $\sim s_{1} t_{1}$.

Now, we prove the continuity of multiplication. Assume that $\left(a_{n}, b_{n}\right)$ converges to $(a, b)$. Since $d_{x, F}\left(a_{n}, a\right)+d_{x, F}\left(b_{n}, b\right) \rightarrow 0$, for each $\varepsilon>0$ there exists a natural number $m$ such that for every natural number $n>m$, we have $d_{x, F}\left(a_{n}, a\right)+d_{x, F}\left(b_{n}, b\right) \leq x_{k}<\varepsilon$ where $x_{k}$ is the greatest term of the sequence satisfying the inequality. Therefore, we get $a_{n} z=a z$ and $b_{n} z=b z$ for all $z \in F_{k}$. It follows that we have

$$
\left(a_{n} b_{n}\right) z=a_{n}\left(b_{n} z\right)=a_{n}(b z)=\left(a_{n} z\right) b=(a z) b=(a b) z
$$

for all $z \in F_{k}$ by the commutative property of multiplication. Thus,

$$
d_{x, F}\left(a_{n} b_{n}, a b\right) \leq x_{k}<\varepsilon .
$$

Hence, $d_{x, F}^{*}\left(\left[a_{n}\right]\left[b_{n}\right],[a][b]\right) \rightarrow 0$ and $\cdot$ is continuous.

We have to work with $\mathbb{Z}_{n}^{\mathbb{Z}}$ instead of $\mathbb{Z}_{n}^{\mathbb{N}}$ if we want to say that the shift map $T$ on the compact metric space is a homeomorphism. Therefore, let us define a compact metric space on the commutative ring $\mathbb{Z}_{n}^{\mathbb{Z}}$ using the notion of filtration.

(i) Let $n$ be a prime number.

The metric space $\left(\mathbb{Z}_{n}^{\mathbb{Z}}, d_{x, F}\right)$ is generated by the filtration

$$
F: F_{0}=\{\theta\} \subseteq F_{1} \subseteq \cdots \subseteq F_{m} \subseteq \cdots
$$

where

$$
F_{m}=\cdots \times\{0\}_{-m} \times\left(\mathbb{Z}_{n}\right)_{-m+1} \times \cdots \times\left(\mathbb{Z}_{n}\right)_{m-1} \times\{0\}_{m} \times \cdots
$$

for each $m \in \mathbb{N}$ and $\theta=(\ldots, 0,0, \ldots)$.

Since

$$
\begin{gathered}
B(s, \varepsilon)=\left\{y \in \mathbb{Z}_{2}^{\mathbb{Z}}: d_{x, F}(s, y)<\varepsilon\right\}= \\
\cdots \times\left(\mathbb{Z}_{2}\right)_{-m} \times\left\{s_{-m+1}\right\} \times \cdots \times\left\{s_{m-1}\right\} \times\left(\mathbb{Z}_{2}\right)_{m} \times \cdots
\end{gathered}
$$

for $s \in \mathbb{Z}_{2}^{\mathbb{Z}}$ and $\varepsilon>0$, where $m \in \mathbb{N}$ minimum such that $x_{m}<\varepsilon$, the metric space $\left(\mathbb{Z}_{2}^{\mathbb{Z}}, d_{x, F}\right)$ has the same topology as $\left(\{0,1\}^{\mathbb{Z}}, d\right)$ does,

$$
\text { where } d(x, y)=\inf \left\{\frac{1}{k+1}: x_{i}=y_{i} \text { for }|i|<k\right\}
$$

$x, y \in\{0,1\}^{\mathbb{Z}}$ defined by Furstenberg $[3$. 
(ii) Let $n$ be a composite number.

The metric space $\left(\left(\mathbb{Z}_{n}^{\mathbb{Z}}\right)^{*}, d_{x, F}^{*}\right)$ is generated by the filtration

$$
F: F_{0}=\{\theta\} \subseteq F_{1} \subseteq \cdots \subseteq F_{m} \subseteq \cdots
$$

where

$$
F_{m}=\cdots \times\{0\}_{-m} \times\{0, a\}_{-m+1} \times \cdots \times\{0, a\}_{m-1} \times\{0\}_{m} \times \cdots
$$

for each $m \in \mathbb{N}, \theta=(\ldots, 0,0, \ldots)$ and $a$ is the smallest prime divisor of $n$.

Proposition 4. The metric space $\left(\left(\mathbb{Z}_{n}^{\mathbb{Z}}\right)^{*}, d_{x, F}^{*}\right)$ defined above is compact.

Proof. It is easy to prove that $\left(\left(\mathbb{Z}_{n}^{\mathbb{Z}}\right)^{*}, d_{x, F}^{*}\right)$ is a sequentially compact space since $\mathbb{Z}_{n}$ is finite set. It is sufficient to show that any sequence $\left(a_{n}\right)_{n \in \mathbb{N}}$ of members of $\left(\mathbb{Z}_{n}^{\mathbb{Z}}\right)^{*}$ has a subsequence converging to an element of $\left(\mathbb{Z}_{n}^{\mathbb{Z}}\right)^{*}$.

Let $a_{n}:=\left[\left(c_{n, m}\right)_{m \in \mathbb{Z}}\right]$ be a sequence in $\left(\mathbb{Z}_{n}^{\mathbb{Z}}\right)^{*}$. Take $\alpha_{0} \in \mathbb{Z}_{n}$ such that $I(0)=$ $\left\{n \in \mathbb{N}: c_{n, 0}=\alpha_{0}\right\}$ is infinite. Let $s(0)$ be the minimum element (or any) of $I(0)$. Now, take $\alpha_{1} \in \mathbb{Z}_{n}$ such that $I(1)=\left\{n \in I(0): c_{n, 1}=\alpha_{1}\right\}$ is infinite. Let $s(1)$ be any element of $I(1)$ such that $s(1)>s(0)$. Then, take $\alpha_{-1} \in \mathbb{Z}_{n}$ such that $I(-1)=\left\{n \in I(1): c_{n,-1}=\alpha_{-1}\right\}$ is infinite and choose $s(-1)$ to be any element of $I(-1)$ such that $s(-1)>s(1)$. Continuing in this way, we obtain an element $a:=\left[\left(\alpha_{m}\right)_{m \in \mathbb{Z}}\right]$ of $\left(\mathbb{Z}_{n}^{\mathbb{Z}}\right)^{*}$. Besides, $\left(a_{s(n)}\right)_{n \in \mathbb{Z}}$ is a subsequence of $\left(a_{n}\right)_{n \in \mathbb{N}}$ that converges to $a$. Consequently, the metric space $\left(\left(\mathbb{Z}_{n}^{\mathbb{Z}}\right)^{*}, d_{x, F}^{*}\right)$ is compact since it is known that a sequentially compact metric space is also compact.

Definition 1. [3] Let $(X, T)$ be a dynamical system. A point $x \in X$ is a recurrent point of $(X, T)$ if for some sequence $n_{k} \rightarrow \infty, T^{n_{k}} x \rightarrow x$.

Let us recall the Birkhoff Multiple Recurrence Theorem. G. D. Birkhoff showed that if $X$ is a compact topological space and $T$ is a continuous map from $X$ to itself, then $X$ has a recurrent point [1] which is called the Birkhoff Recurence Theorem. The following theorem, due to Furstenberg [3], generalizes the Birkhoff Recurrence Theorem since it guarantees the existence of a point which is simultaneously recurrent for $T, T^{2}, \ldots, T^{n} \quad n \geq 1$.

Theorem 2 (Birkhoff Multiple Recurrence). [3] Let $X$ be a compact metric space, and let $T: X \rightarrow X$ be a continuous map. Then for any integer $r \geq 1$, there exists a point $x \in X$ and a sequence $n_{k} \rightarrow \infty$ with $T^{n_{k}} x \rightarrow x, T^{2 n_{k}} x \rightarrow x, \ldots, T^{r n_{k}} x \rightarrow x$.

Definition 2. An arithmetic progression of length $l$ is a sequence of integers of the form

$$
a, a+d, a+2 d, \ldots, a+(l-1) d
$$

where $d \neq 0$. 
Now, we give a variant of the proof of Van der Waerden's theorem by Furstenberg 3 .

Theorem 3 (Van der Waerden). Let $\mathbb{Z}=\bigcup_{i=0}^{r-1} C_{i}$ be a partition of the integers into $r$ subsets. Then one of the sets $C_{j}$ contains an arithmetic progression of length $l$.

Proof. Let $\mathbb{Z}=\bigcup_{i=0}^{r-1} C_{i}$ be a partition of the integers.

Case 1 Suppose $r$ is a composite number. Let us define $\xi \in \mathbb{Z}_{r}^{\mathbb{Z}}$ which corresponds to a partition of $\mathbb{Z}$ with $r$ sets. In other words,

$$
\mathbb{Z}=\cup C_{i} \text { where } C_{i}=\left\{n: \xi_{n}=i\right\}
$$

represents an equivanlance class partition of $\mathbb{Z}$ in $\Omega:=\left(\left(\mathbb{Z}_{r}^{\mathbb{Z}}\right)^{*}, d_{x, F}^{*}\right)$. Let $X \subseteq \Omega$,

$$
X=\overline{\left\{T^{n}[\xi], n \in \mathbb{Z}\right\}}
$$

be the closure of the set of all translates of $[\xi]$. According to the Birkhoff Multiple Recurrence theorem, there exists $[\beta] \in X$ and an $n>0$ with the points $[\beta], T^{n}[\beta], \ldots, T^{l n}[\beta]$ within distance less than $x_{1}$ of one another. We know that for two elements $[\alpha]$ and $[\gamma]$ in $\Omega, d_{x, F}^{*}([\alpha],[\gamma])<x_{1}$ implies that one of

$$
\alpha_{0}=\gamma_{0}, \alpha_{0}=\gamma_{0}+q, \ldots, \alpha_{0}=\gamma_{0}+(a-1) q
$$

is satisfied in $\mathbb{Z}_{r}$ by virtue of the definition of $d_{x, F}^{*}$ where $r=a q$ and $a$ is the smallest prime divisor of $r$. It follows that

$$
\begin{aligned}
& \beta_{0}=\beta_{k_{11} \cdot n}=\beta_{k_{12} \cdot n}=\cdots=\beta_{k_{1 S_{1}} \cdot n} \\
& \beta_{0}+q=\beta_{k_{21} \cdot n}=\beta_{k_{22} \cdot n}=\cdots=\beta_{k_{2 S_{2}} \cdot n} \\
& \beta_{0}+(a-1) q=\beta_{k_{a 1} \cdot n}=\beta_{k_{a 2} \cdot n}=\cdots=\beta_{k_{a S_{a}} \cdot n}
\end{aligned}
$$

such that $\{1,2, \ldots, l\}=\bigcup_{i=1}^{a}\left\{k_{i 1}, \ldots, k_{i S_{i}}\right\}$.

Since $[\beta] \in X=\overline{\left\{T^{j}[\xi], j \in \mathbb{Z}\right\}}$, we can find $m$ so that

$$
\begin{gathered}
\xi_{m}=\xi_{m+k_{11} \cdot n}=\xi_{m+k_{12} \cdot n}=\cdots=\xi_{m+k_{1 S_{1} \cdot n}} \\
\xi_{m}+q=\xi_{m+k_{21} \cdot n}=\xi_{m+k_{22} \cdot n}=\cdots=\xi_{m+k_{2 S_{2} \cdot n}} \\
\vdots \\
\xi_{m}+(a-1) q=\xi_{m+k_{a 1} \cdot n}=\xi_{m+k_{a 2} \cdot n}=\cdots=\xi_{m+k_{a S_{a} \cdot n} .} .
\end{gathered}
$$

Then one of $C_{i} \cup C_{i+q} \cup \ldots \cup C_{i+(a-1) q}$, where $i \in\{0,1, \ldots, q-1\}$, contains an arithmetic progression of length $l$.

Now, let us consider the partition 
$\mathbb{Z}=\bigcup_{i=0}^{a r-1} D_{i}$ where $C_{i}=D_{i} \cup D_{i+r} \cup \ldots \cup D_{i+(a-1) r}$ and $i \in\{0,1, \ldots, r-1\}$.

If we apply the above method for the partition

$$
\mathbb{Z}=\bigcup_{i=0}^{a r-1} D_{i} \text { and } \Omega:=\left(\left(\mathbb{Z}_{a r}^{\mathbb{Z}}\right)^{*}, d_{x, F}^{*}\right),
$$

we obtain that one of $C_{i}=D_{i} \cup D_{i+r} \cup \ldots \cup D_{i+(a-1) r}$, where $i \in\{0,1, \ldots, r-1\}$, contains an arithmetic progression of length $l$.

Case 2 Let $r$ be a prime number. Now, let us arrange the partition $\mathbb{Z}=\bigcup_{i=0}^{r-1} C_{i}$ in order to turn into the first case. If we take $r^{\prime}=2 r$ and

$$
\mathbb{Z}=\bigcup_{i=0}^{r^{\prime}-1} E_{i} \text { where } C_{i}=E_{i} \cup E_{i+r} \text { for } i \in\{0,1, \ldots, r-1\}
$$

it follows from Case 1 that one of $E_{i}$ contains an arithmetic progression of length $l$.

Author Contributions Statements The authors jointly worked on the results and they read and approved the final manuscript.

Declaration of Competing Interests The authors declare that they have no competing interest.

Acknowledgement The authors are very thankful to Haydar Göral for his helpful comments when preparing this paper. Also, the authors would like to thank the referees for their valuable comments which helped to improve the manuscript.

\section{REFERENCES}

[1] Birkhoff, G.D., Dynamical Systems, Math. Soc. Coll. Publ., vol 9, Amer. Math. Soc., Providence RI, 1927. https://doi.org/http://dx.doi.org/10.1090/coll/009

[2] Engelking, R., General Topology, Second Edition, Heldermann Verlag, Berlin, 1989.

[3] Furstenberg, H., Poincare recurrence and number theory, Bull. of the A. Math. Soc., 5 (3) (1981), 211-234.

[4] Furstenberg, H., Recurrence in Ergodic Theory and Combinatorial Number, Princeton University Press, Princeton, New Jersey, 1981.

[5] Van der Waerden, B.L., Beweis einer baudetschen vermutung, Nieuw Arch. Wisk., 15 (1927), $212-216$. 